\title{
Hall-Petch Relationship and Dislocation Model for Deformation of Ultrafine-Grained and Nanocrystalline Metals
}

\author{
Masaharu Kato* \\ Department of Materials Science and Engineering, Interdisciplinary Graduate School of Science and Engineering, \\ Tokyo Institute of Technology, Yokohama 226-8502, Japan
}

Models and theories to explain the Hall-Petch relationship are reviewed briefly. Then, a dislocation model to incorporate some characteristic mechanical properties of ultrafine-grained and nanocrystalline metals will be introduced and used to explain some experimental results. The model is based on the idea that dislocations emitted from grain boundaries and bow out into grain interiors during their propagation are responsible for plastic deformation and thermally-activated depinning process at grain boundaries is regarded to be rate controlling. Some implications of the model are discussed in the light of recent experimental results. [doi:10.2320/matertrans.MA201310]

(Received July 31, 2013; Accepted August 26, 2013; Published December 25, 2013)

Keywords: Hall-Petch relationship, yield stress, dislocation, grain boundary, strength, ultrafine-grained metal, strain rate, temperature, thermal activation

\section{Introduction}

It is well known that ultrafine-grained (UFG) and nanocrystalline (NC) metals and alloys show characteristic mechanical properties different from those of conventional coarse-grained (CG) counterparts. ${ }^{1-6)}$ For example, as grain size becomes smaller, (1) strength becomes higher (the HallPetch (HP) behavior) and then lower (the inverse HP behavior). (2) strength of fcc metals becomes more sensitive to temperature and strain rate. (3) grain boundaries (GBs) play more important roles on plastic deformation. A theory on the mechanical properties of UFG and NC materials should reasonably explain these characteristics.

For the purpose of this special issue and in order to understand the general role of GBs in the strength of materials, several representative models to explain the HP relationship will be reviewed briefly in the present study. Then, a dislocation model to incorporate the above characteristic mechanical properties of UFG and NC metals will be introduced and used to explain some experimental results.

\section{Representative Models and Theories of the Hall- Petch Relationship}

Following the pioneering work by Hall ${ }^{7)}$ and Petch, ${ }^{8)}$ many experimental and theoretical studies ${ }^{2,9-16)}$ have been conducted to show the linear relationship between yield or flow stress and inverse square root of grain size.

$$
\sigma=\sigma_{0}+k_{\mathrm{HP}} d^{-1 / 2}
$$

where $\sigma$ is the yield or flow stress, $\sigma_{0}$ the friction stress, $k_{\mathrm{HP}}$ the Hall-Petch coefficient and $d$ the grain size. Although main focus in recent studies may be placed on the behavior of UFG and NC materials including the so-called HP breakdown or inverse HP phenomena, only models to explain the classic HP law of eq. (1) will be reviewed in this study.

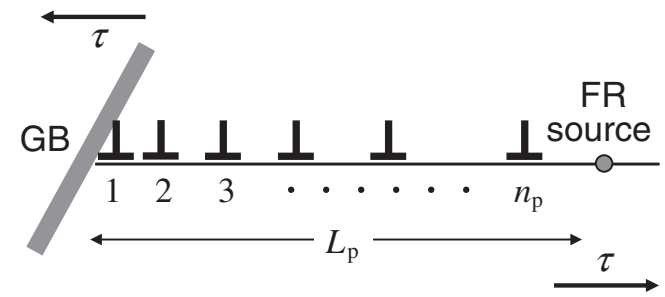

Fig. 1 Dislocations generated from a Frank-Read (FR) source pile up at a grain boundary (GB). $L_{\mathrm{p}}$ is the pile-up length and $n_{\mathrm{p}}$ is the number of pileup dislocations.

\subsection{Dislocation pile-up model}

This model was adopted by both Hall and Petch in their original papers ${ }^{7,8)}$ and has been the most commonly quoted model ever since. It is based on the idea of dislocation pile up at a $G B,{ }^{17)}$ as shown in Fig. 1 . The pile-up length $L_{\mathrm{p}}$ and the number of dislocations $n_{\mathrm{p}}$ are related to each other as ${ }^{18,19)}$

$$
L_{\mathrm{p}}=n_{\mathrm{p}} \mu b / A \tau_{\mathrm{e}},
$$

where $\mu$ is the shear modulus, $b$ the magnitude of the Burgers vector, $A$ a dimensionless constant of about two and $\tau_{\mathrm{e}}$ is a part of the applied shear stress $\tau$ which contributes to the dislocation pile up. It is known that the stress concentration at the pile-up front is $\tau_{\mathrm{e}}$ times the number of piled-up dislocations $n_{\mathrm{p}}$, i.e., $n_{\mathrm{p}} \tau_{\mathrm{e}}{ }^{18,19)}$ If this stress concentration reaches a critical value $\tau_{\mathrm{c}}$ which is enough for the nucleation of a new dislocation in the adjacent grain, yielding of the polycrystal is considered to occur. The condition of the yielding thus becomes

$$
\tau_{\mathrm{c}}=n_{\mathrm{p}} \tau_{\mathrm{e}}
$$

With eqs. (2) and (3) together with the assignment of $L_{\mathrm{p}}=$ $d / 2$ and $\tau=\tau_{0}+\tau_{\mathrm{e}}$ where $\tau_{0}$ is the friction shear stress, we have

$$
\tau_{\mathrm{c}} \approx \tau_{\mathrm{e}}^{2} d / \mu b
$$

and

$$
\tau=\tau_{0}+\tau_{\mathrm{e}}=\tau_{0}+\left(\tau_{\mathrm{c}} \mu b\right)^{1 / 2} d^{-1 / 2} .
$$




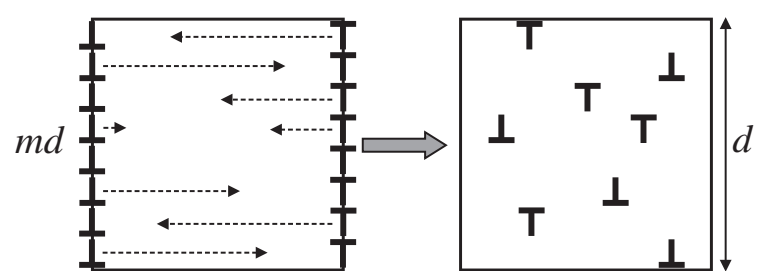

Fig. 2 Dislocations generated from GB ledges move and form dislocation forests in a grain.

Therefore, we arrive at

$$
\begin{aligned}
& \sigma=\sigma_{0}+\sigma_{\mathrm{e}}=M\left(\tau_{0}+\tau_{\mathrm{e}}\right)=\sigma_{0}+k_{\mathrm{HP}} d^{-1 / 2} \\
& \text { with } k_{\mathrm{HP}}=M\left(\tau_{\mathrm{c}} \mu b\right)^{1 / 2},
\end{aligned}
$$

where $M$ is the Taylor orientation factor, $\sigma=M \tau, \sigma_{0}=M \tau_{0}$ and $\sigma_{\mathrm{e}}=M \tau_{\mathrm{e}}=k_{\mathrm{HP}} d^{-1 / 2}$.

\subsection{Boundary source model}

Although the pile-up model originally developed for iron has been frequently quoted to explain the HP relationship, some concerns of this model in early 1960's were that (1) the Frank-Read source was generally accepted but not very frequently observed, and (2) no dislocation pile-ups were found in iron. These led Li to propose the boundary source model ${ }^{20)} \mathrm{He}$ has considered that dislocations are generated at GB ledges and all these dislocations form dislocation forests in grain interiors.

As shown in Fig. 2, suppose that a grain has a cubic shape with side length $d$ and there are $m$ ledges per unit length of a boundary. Since each GB has two abutting grains and the left and right GBs in Fig. 2 have $m d$ ledges each, they generate $m d$ dislocations of length $d$ per grain. When these dislocations move and form forest dislocations in the grain interior, the forest dislocation density $\rho^{\mathrm{f}}$ becomes

$$
\rho^{\mathrm{f}}=m d^{2} / d^{3}=m / d .
$$

On the other hand, the well-known Taylor equation is written as

$$
\sigma=\sigma_{0}+M \alpha \mu b \sqrt{\rho},
$$

where $\alpha$ is a constant of about 0.3 to 0.5 and $\rho$ the dislocation density. Regarding $\rho^{\mathrm{f}}$ and $\rho$ in eqs. (7) and (8) are the same, we obtain

$$
\sigma=\sigma_{0}+M \alpha \mu b m^{1 / 2} d^{-1 / 2} .
$$

Aside from slight numerical difference, eq. (9) is the same as eq. (7) in Li's paper: ${ }^{20)} \sigma=\sigma_{0}+\alpha \mu b(8 m / \pi)^{1 / 2} d^{-1 / 2}$. In fact, Li compared the magnitude of $\sigma$ in his model with that of the pile-up model and reasonable agreement was found. ${ }^{20)}$ Since no dislocation pile up is needed, the boundary source model has been paid much attention, too.

\subsection{Work hardening model}

The above two models do not contain plastic strain in the formulation. In this sense, the derived HP eqs. (6) and (9) are applicable to the yield stress of polycrystalline materials. On the other hand, models have been developed to explain the HP relationship for flow and fracture stresses. For example, the HP equation at an arbitrary plastic strain can be written $\operatorname{as}^{21)}$

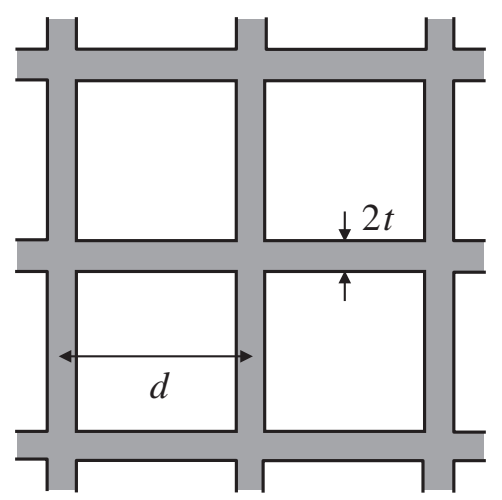

Fig. 3 Cross section of cubic grains of grain size $d$ with work-hardened GB layers of thickness $2 t$.

$$
\sigma(\varepsilon)=\sigma_{0}(\varepsilon)+k_{\mathrm{HP}}(\varepsilon) d^{-1 / 2}
$$

where $\varepsilon$ is tensile (or compressive) plastic strain which is related to the shear plastic strain $\gamma$ as $\gamma=M \varepsilon$.

Meakin and Petch ${ }^{22)}$ assumed that the average slip distance $L^{\mathrm{s}}$ of glide dislocations was comparable to the grain size and the shear plastic strain could be written as

$$
\gamma=\rho b L^{\mathrm{s}} \text {. }
$$

Combining eq. (11) with eq. (8), one readily obtains

$$
\begin{aligned}
\sigma & =\sigma_{0}+M \alpha \mu\{b(\gamma / \beta)\}^{1 / 2} d^{-1 / 2} \\
& =\sigma_{0}+M \alpha \mu\{b(M \varepsilon / \beta)\}^{1 / 2} d^{-1 / 2},
\end{aligned}
$$

by assigning

$$
L^{\mathrm{s}}=\beta d \quad \text { with } \quad 0<\beta \leq 1 .
$$

At a given grain size, this model predicts parabolic work hardening (eq. (12)). Furthermore, at a given plastic strain, the model assumes that the dislocation density is inversely proportional to the grain size, as can be seen from eqs. (11) and (13). In fact, Conrad and co-workers showed that these were really the case. ${ }^{23-25)}$

\subsection{Composite model}

Models belonging to this category consider that a polycrystalline material is made of two regions; grain interiors with lower flow stress $\sigma_{\mathrm{i}}$ and GB work-hardened layers with higher flow stress $\sigma_{\mathrm{b}}{ }^{26-29)}$ When a composite made of cubic grains with grain size $d$ and GB layer thickness $2 t$ are considered, as shown in Fig. 3, the flow stress $\sigma$ of this composite can be written from a simple rule of mixture as

$$
\begin{aligned}
\sigma & =\left\{\frac{(d-2 t)^{2}}{d^{2}}\right\} \sigma_{\mathrm{i}}+\left\{\frac{d^{2}-(d-2 t)^{2}}{d^{2}}\right\} \sigma_{\mathrm{b}} \\
& =\sigma_{\mathrm{i}}+4\left(\sigma_{\mathrm{b}}-\sigma_{\mathrm{i}}\right) \frac{t}{d}-4\left(\sigma_{\mathrm{b}}-\sigma_{\mathrm{i}}\right)\left(\frac{t}{d}\right)^{2} .
\end{aligned}
$$

Meyers and Ashworth ${ }^{29)}$ considered a more detailed geometry for spherical grains to obtain

$$
\sigma=\sigma_{\mathrm{i}}+8\left(\sigma_{\mathrm{b}}-\sigma_{\mathrm{i}}\right) \frac{t}{d}-16\left(\sigma_{\mathrm{b}}-\sigma_{\mathrm{i}}\right)\left(\frac{t}{d}\right)^{2} .
$$

The first and second terms of the right-hand side of eq. (15) are dominant for large grains of $d \gg t$ which is usually the case for coarse-grained materials. Then, not the $d^{-1 / 2}$ 


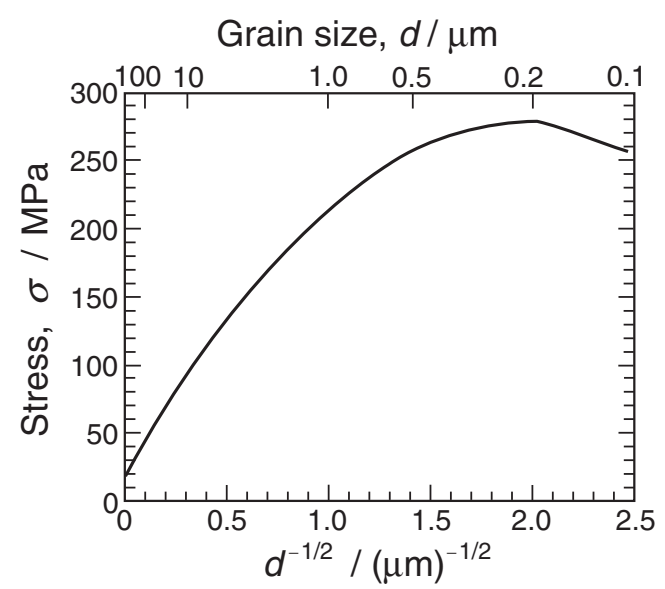

Fig. 4 Relationship between stress $\sigma$ and inverse square root of grain size $d$ predicted from eqs. (15) and (16). Numerical values of $\sigma_{\mathrm{i}}=20 \mathrm{MPa}$, $\sigma_{\mathrm{b}}=280 \mathrm{MPa}$ and $\xi=0.125 \mu \mathrm{m}^{1 / 2}$ were chosen.

dependence but the $d^{-1}$ dependence of the flow stress is naturally expected from the composite model.

For the composite model to explain the HP relationship, it is necessary to assume that GB layer thickness $2 t$ is an increasing function of grain size such as ${ }^{29)}$

$$
t=\xi d^{1 / 2}
$$

where $\xi$ is an adjustable parameter. This assumption may be justified since larger grains can accumulate more dislocations in work-hardened GB layers so that the layer thickness increases with increase in grain size. Then, from eqs. (15) and (16), we find that $d^{-1 / 2}$ dependence of $\sigma$ is satisfied for larger grain size and as grain size becomes smaller, the slope of the curve becomes smaller, as shown in Fig. 4.

\subsection{Dislocation density model}

This model is based on the idea that dislocations in materials are divided into two categories; statistically stored (SS) dislocations and geometrically necessary (GN) ones. ${ }^{30)}$ Uniform deformation accumulates SS dislocations in grain interiors whereas non-uniform deformation creates GN dislocations to accommodate material overlaps or voids at GBs.

Suppose applied shear stress $\tau$ has induced plastic strain $\gamma$ in the left and right grains and no plastic strain in the center grain, as schematically shown in Fig. 5. The SS dislocation density is expressed, similar to eq. (11), as

$$
\rho^{\mathrm{s}}=\frac{C_{1} \gamma}{b L^{\mathrm{s}}}=M \frac{C_{1} \varepsilon}{b L^{\mathrm{s}}},
$$

where $C_{1}$ is a constant depending on the shape, orientation and distribution of grains. Equation (17) indicates that the SS dislocation density is independent of the grain size. On the other hand, the number of GN dislocations $n_{\mathrm{g}}$ near the GBs of the center grain can be written as ${ }^{30 \text { ) }}$

$$
n_{\mathrm{g}}=C_{2} \gamma d / b,
$$

where $C_{2}$ is another geometrical constant. Therefore, GN dislocation density in the center grain becomes ${ }^{30)}$

$$
\rho^{\mathrm{g}}=\frac{n_{\mathrm{g}} d}{d^{3}}=\frac{C_{2} \gamma}{b d}=M \frac{C_{2} \varepsilon}{b d} .
$$

(a)

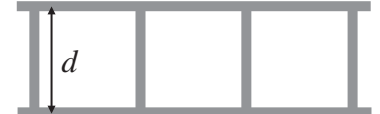

(b)

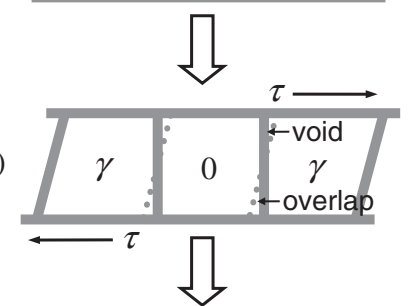

(c)

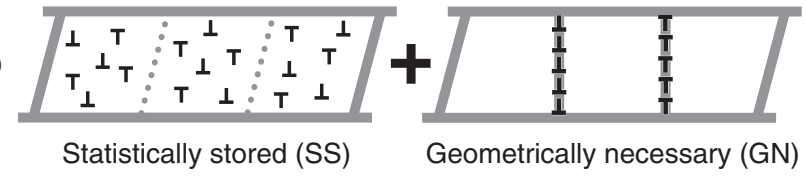

Fig. 5 (a) Deforming three cubic grains. (b) If the plastic strain of the center grain happens to be smaller than that of the other two grains, voids and overlaps are created at the GBs of the center grain. (c) Uniform deformation $\gamma$ creates statistically stored (SS) dislocations while nonuniform deformation causes generation of geometrically necessary (GN) dislocations to accommodate the voids and overlaps.

From eqs. (8), (17) and (19), the flow stress of the dislocation density model becomes ${ }^{31)}$

$$
\sigma(\varepsilon)=\sigma_{0}+M^{3 / 2} \alpha \mu b \sqrt{\varepsilon}\left(\frac{C_{1}}{b L^{\mathrm{s}}}+\frac{C_{2}}{b d}\right)^{1 / 2} .
$$

If $\mathrm{GB}$ contribution dominates, GN dislocations govern the strength and the above equation reduces to the HP relationship:

$$
\sigma(\varepsilon)=\sigma_{0}+M^{3 / 2} \alpha \mu \sqrt{C_{2} b \varepsilon} d^{-1 / 2} .
$$

This equation has been compared with the flow stress of many metals and satisfactory agreement was found. ${ }^{32,33)}$

Ashby's idea of SS and GN dislocations has led to the establishment of so-called strain-gradient plasticity. ${ }^{34-39)}$ With this approach, a length scale is introduced in the classic size-independent plasticity theory. Since GN dislocations are associated with non-uniform deformation, they can be connected with local gradient of strain, i.e.,

$$
\rho^{\mathrm{g}}=\frac{1}{b} \frac{\partial \gamma}{\partial l} \equiv \frac{\eta}{b}
$$

where $l$ is a length scale and $\eta$ the strain gradient. ${ }^{34-39)}$ If the length scale can be taken proportional to grain size $d$ so that $l=C_{3} d\left(0<C_{3} \leq 1\right)$, and when the average strain gradient over a grain is expressed as $\eta=\Delta \gamma / C_{3} d(\Delta \gamma$ : strain amplitude over the length $l$ ), eq. (22) becomes

$$
\rho^{\mathrm{g}}=\frac{\Delta \gamma}{C_{3} b d} .
$$

This is similar to eq. (19) showing that GN dislocation density is inversely proportional to grain size. Therefore, the HP type $d^{-1 / 2}$ dependence of flow stress can be obtained from eqs. (8) and (23).

Though the idea of $\mathrm{SN}$ and GN dislocations and the discussion based on the scale-dependent plasticity are very useful, it cannot be applied to the initial yield stress of wellannealed materials since SS and GN dislocations are not accumulated before the onset of plastic deformation. 


\subsection{Thermally-activated deformation processes}

To explain the temperature and strain-rate dependence of yield and flow stresses of polycrystals, thermally-activated deformation processes have been discussed in conjunction with the HP equation. Using the pile-up model and differentiating both sides of eq. (6) with respect to $\ln \dot{\varepsilon}$ where $\dot{\varepsilon}$ is the tensile (compressive) strain rate, we have

$$
\frac{\partial \sigma}{\partial \ln \dot{\varepsilon}}=\frac{\partial \sigma_{0}}{\partial \ln \dot{\varepsilon}}+M(\mu b)^{1 / 2} \frac{\partial \tau_{\mathrm{c}}^{1 / 2}}{\partial \ln \dot{\varepsilon}} d^{-1 / 2}
$$

On the other hand, activation volume is generally defined as

$$
v^{*} \equiv k_{\mathrm{B}} T\left(\frac{\partial \ln \dot{\gamma}}{\partial \tau}\right)=M k_{\mathrm{B}} T\left(\frac{\partial \ln \dot{\varepsilon}}{\partial \sigma}\right),
$$

where $k_{\mathrm{B}}=1.381 \times 10^{-23} \mathrm{~J} \mathrm{~K}^{-1}$ is the Boltzmann constant. From eqs. (24) and (25), we arrive at the following expression $^{16,40-42)}$

$$
\frac{1}{v^{*}} \equiv \frac{1}{v_{0}^{*}}+\left(\frac{k_{\mathrm{HP}}}{2 M \tau_{\mathrm{c}} v_{\mathrm{c}}^{*}}\right) d^{-1 / 2},
$$

where $v_{0}^{*} \equiv M k_{\mathrm{B}} T\left(\partial \ln \dot{\varepsilon} / \partial \sigma_{0}\right)$ represents thermally-activated deformation in grain interiors and $v_{\mathrm{c}}^{*} \equiv M k_{\mathrm{B}} T\left(\partial \ln \dot{\varepsilon} / \partial \sigma_{\mathrm{c}}\right)$ with $M \tau_{\mathrm{c}}=\sigma_{\mathrm{c}}$ is for the GB region. When the term $\tau_{\mathrm{c}} v_{\mathrm{c}}^{*}$ is constant, eq. (26) predicts the HP-type relationship between $v^{*-1}$ and $d$. In fact, from experimentally found such HP behavior in fcc polycrystals, Armstrong and co-workers have interpreted that $v_{0}^{*}$ values represent the in-grain dislocation intersection with forest dislocations and $v_{\mathrm{c}}^{*}$ values represent cross slip enhanced by stress concentration near $\mathrm{GBs}^{16,40-42)}$.

For UFG metals, Conrad and co-workers have also conducted thermal activation analysis ${ }^{43-47)}$ and essentially the same equation as eq. (26) has been obtained. ${ }^{14)}$ They have concluded that deformation kinetics of UFG metals with $d$ between 10 and $100 \mathrm{~nm}$ (Regime II in their studies) is controlled by GB shear promoted by the dislocation pile-up.

It appears that despite the fact that dislocation pile-ups are not necessarily observed, the pile-up model has been used most extensively, in particular to discuss thermally-activated deformation processes in polycrystals.

\section{Deformation Kinetics of UFG and NC Materials}

As mentioned in Introduction, discussion on thermallyactivated deformation kinetics is indispensable to understand mechanical properties of UFG and NC materials. Furthermore, for these materials, many studies have pointed out that partial or perfect dislocations emitted from GBs play essential roles in the plastic deformation. ${ }^{48-58)}$ Some investigators believe that dislocations once nucleated at a GB would cross the grain rapidly without stopping in a grain. ${ }^{51,59,60)}$ On the other hand, it has been pointed out that as a propagating dislocation traverses the grain towards the opposite boundary, its two ends may be pinned by GB impurities or ledges. ${ }^{56,61,62)}$ When such pinning occurs, the depinning process can be a rate-controlling step of the thermallyactivated deformation of UFG materials. ${ }^{56-58,61-63)}$

Using the simplified geometry shown in Fig. 6, Kato analyzed the thermally-activated depinning process at a GB as a glide dislocation bows out into a grain. ${ }^{58)}$ Later, some

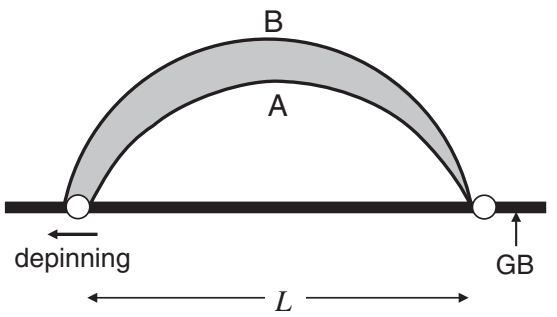

Fig. 6 Schematic of dislocation depinning at a GB. Bowing-out dislocation A under applied stress is pinned by two GB pinning points of separation $L$. If thermally-activated depinning occurs at one of the two GB pinning points, dislocation A changes into dislocation B keeping the same radius of curvature. The area surrounded by the two dislocations A and B is the activation area.

investigators have found that experimentally observed rate parameters, such as activation volume and strain-rate sensitivity for fcc UFG and NC metals, can be explained reasonably by this model. ${ }^{64-67)}$

Analytical formulation of the dislocation bow-out and depinning model is summarized as follows. ${ }^{57,58)}$ The yield stress $\sigma_{\mathrm{y}}$ of a UFG or NG material consists of athermal $\left(\sigma_{\mathrm{a}}\right)$ and thermal $\left(\sigma^{*}\right)$ stress components as

$$
\sigma_{\mathrm{y}}=\sigma_{\mathrm{a}}+\sigma^{*}=M\left(\tau_{\mathrm{a}}+\tau^{*}\right) .
$$

Here, the athermal component is written as

$$
\tau_{\mathrm{a}}=\frac{\mu b}{2 \pi d} \ln \left(\frac{d}{10 b}\right),
$$

and the thermal component as

$$
\tau^{*}=\tau_{\mathrm{m}}\left[1-\left\{\frac{k_{\mathrm{B}} T \ln \left(\dot{\gamma}_{0} / \dot{\gamma}\right)}{G_{0}}\right\}^{2 / 3}\right],
$$

with

$$
\tau_{\mathrm{m}}=\frac{3 \mu b}{2 \pi(d+2 \lambda)} \ln \left(\frac{d+2 \lambda}{30 b}\right)
$$

and

$$
\dot{\gamma}=\dot{\gamma}_{0} \exp \left[-\frac{G\left(\tau^{*}\right)}{k_{\mathrm{B}} T}\right] .
$$

In the above equations, $\dot{\gamma}$ is the shear strain rate, $G\left(\tau^{*}\right)$ and $G_{0}$ are the activation energies with and without $\tau^{*}$, respectively, $\lambda$ the minimum GB source length of dislocations that emit and bow out from GBs and $\dot{\gamma}_{0}=1 \times 10^{7} \mathrm{~s}^{-1}$ is assumed. $^{58)}$

Geometrically-derived activation volume $v_{\mathrm{d}}^{*}$ of the depinning process in Fig. 6 can be obtained as ${ }^{58)}$

$$
\begin{aligned}
v_{\mathrm{d}}^{*}= & r^{2} b \sin ^{-1}\left(\frac{L+w^{*}}{2 r}\right)-\left(\frac{L+w^{*}}{2}\right) \sqrt{r^{2}-\left(\frac{L+w^{*}}{2}\right)^{2}} \\
& -\left[r^{2} b \sin ^{-1}\left(\frac{L}{2 r}\right)-\left(\frac{L}{2}\right) \sqrt{r^{2}-\left(\frac{L}{2}\right)^{2}}\right]
\end{aligned}
$$

where $L$ is the distance between the pinning points,

$$
r=\frac{\mu b}{4 \pi \tau^{*}} \ln \left(\frac{L}{10 b}\right)
$$




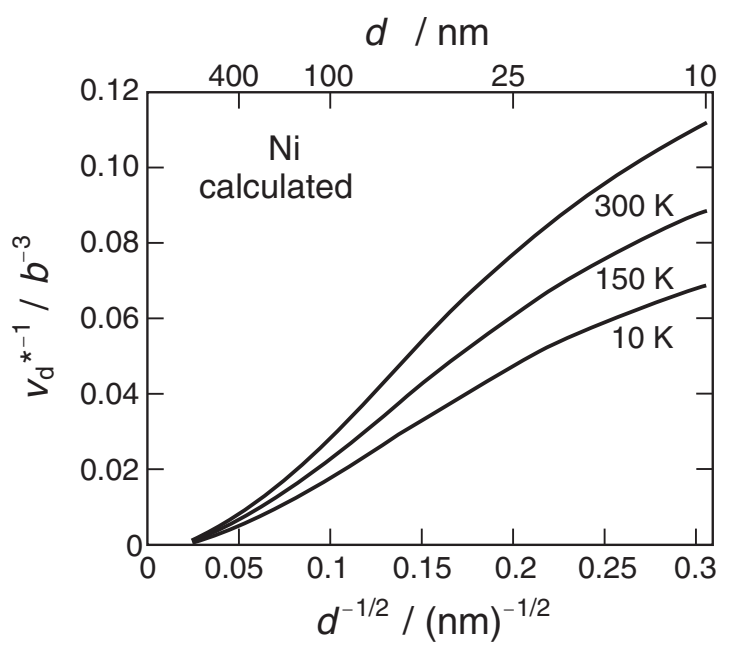

Fig. 7 Calculated relationship between the reciprocal activation volume $v_{\mathrm{d}}^{*-1}$ and square root of grain size $d$ by using the bow-out and depinning model $^{58)}$ with numerical values of Ni. Note that near HP relationship holds between $v_{\mathrm{d}}^{*-1}$ and $d$ and that the activation volume is a decreasing function of temperature.

is the radius of curvature for the bowing-out dislocation and

$$
w^{*}=b\left\{1-\left(\tau^{*} / \tau_{\mathrm{m}}\right)\right\}^{1 / 2}
$$

is the activation distance.

Since this model is already known to predict the HP-type relationship between yield stress and grain size at a chosen deformation temperature, ${ }^{57,58)}$ some new implications of the model relating to the thermally-activated deformation process will be discussed below. First, let us examine whether the reciprocal of the activation volume defined in eq. (32) shows the HP-type behavior with respect to the grain size. For this purpose, numerical values for $\mathrm{Ni}$ will be chosen as in the previous study: ${ }^{58)} \mu=79 \mathrm{GPa}$, $b=0.249 \mathrm{~nm}, \quad G_{0}=2.0 \mathrm{eV} \quad\left(=3.20 \times 10^{-19} \mathrm{~J}\right), \quad \dot{\gamma}=1 \times$ $10^{-4} \mathrm{~s}^{-1}, \quad \dot{\gamma}_{\mathrm{o}}=1 \times 10^{7} \mathrm{~s}^{-1}, \quad L=(d+2 \lambda) / 3 \quad$ and $\lambda=$ $10 \mathrm{~nm}$. Figure 7 shows the relationship between $v_{\mathrm{d}}^{*-1}$ and $d^{-1 / 2}$ calculated from eq. (32). Three curves are for three different temperatures; 10,150 and $300 \mathrm{~K}$. It can be seen that the curves are nearly straight, in particular, when $d$ is larger than about $25 \mathrm{~nm}$. Therefore, the HP-like behavior, as discussed in section 2.6, approximately holds between the reciprocal activation volume and grain size in Kato's model, too.

It should be noted from Fig. 7 that for a given grain size, $v_{\mathrm{d}}^{*-1}$ is an increasing function or $v_{\mathrm{d}}^{*}$ is a decreasing function of temperature. This unique and anomalous behavior, different from that for coarse-grained polycrystals, has been noted $^{14,45,56)}$ and some researchers discussed it using Kato's model. ${ }^{64-67)}$

Relating to the above anomalous temperature dependence of activation volume, Conrad ${ }^{14,45)}$ and Conrad and Yang ${ }^{68)}$ have shown for their regime II of the grain size $(d \approx$ $10-1000 \mathrm{~nm}$ ) that the activation volume $v^{*}$ becomes an increasing function of the applied stress. According to them, this anomalous stress dependence of $v^{*}$ is also consistent with their proposed rate-controlling process for fcc UFG materials

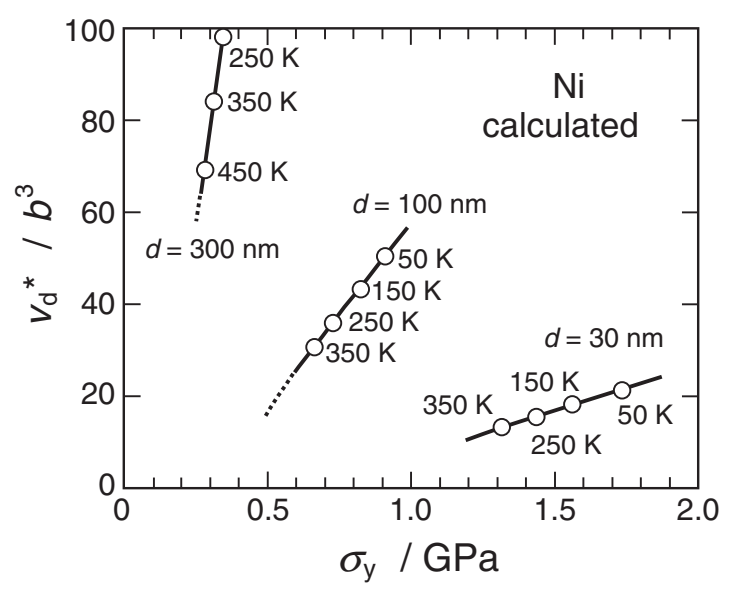

Fig. 8 Stress dependence of the activation volume $v_{\mathrm{d}}^{*}$ predicted by the bow-out and depinning model ${ }^{58)}$ with numerical values of $\mathrm{Ni}$ for three grain sizes. At a fixed grain size, the yield stress $\sigma_{\mathrm{y}}$ is calculated as a function of temperature. Note that anomalous positive stress dependence found by Conrad et al. ${ }^{14,45-47,68)}$ can be reproduced by the present model.

in regime II, i.e., GB shear promoted by the pile-up of dislocations. ${ }^{14,45-47,68)}$ Kapoor and Chakravartty have also found the anomalous stress dependence of $v^{*}$ from an experiment using UFG Al-Mg alloy. ${ }^{69)}$ They pointed out that dislocation-solute interaction may be a possible origin of this $v^{*}-\sigma$ behavior.

The relationship between $v_{\mathrm{d}}^{*}$ and $\sigma_{\mathrm{y}}$ can also be derived from the present bow-out and depinning model since $v_{\mathrm{d}}^{*}$ can be expressed as a function of $\tau^{*}$ from eqs. (29), (32) and (33). The calculated results are shown in Fig. 8. Indeed, we find that $v_{\mathrm{d}}^{*}$ becomes an increasing function of $\sigma_{\mathrm{y}}$ $\left(=M\left(\tau_{\mathrm{a}}+\tau^{*}\right)\right)$. It is encouraging to know that not only the $v_{\mathrm{d}}^{*}-\sigma_{\mathrm{y}}$ relationship but also the predicted values of $v_{\mathrm{d}}^{*}$ are in quantitative agreement with experimental data obtained for $\mathrm{Ni}^{56)}$ In conclusion, we can say that the present model of dislocation bow-out and depinning can explain many observed characteristics of deformation kinetics of fcc UFG and NC materials.

\section{Concluding Remarks}

This paper consists of two parts. In the first part, several representative models to explain the HP relationship were reviewed. In the second part, recently developed dislocation bow-out and depinning model has been used to explain some aspects of deformation kinetics in UFG and NC materials together with the HP-like behavior of reciprocal activation volume as a function of grain size.

Although it appears that the dislocation pile-up model is still the most frequently adopted one to explain the HP relationship, the other models have their own merit and advantages. Furthermore, seeing the fact that the HP relationship applies not only to yield stress but also to flow and fracture stresses and even to fatigue and creep, ${ }^{70)}$ it appears that the HP-type size dependence of strength is a rather general result of natural dislocation behavior. Revealing various aspects of physics behind the HP law certainly remains to be an attractive and challenging research subject. 


\section{Acknowledgement}

This study was supported by a Grant-in-Aid for Scientific Research on Innovative Area "Bulk Nanostructured Metals" through the Ministry of Education, Culture, Sports, Science and Technology, Japan (contract No. 22102006).

\section{REFERENCES}

1) Q. Wei, S. Cheng, K. T. Ramesh and E. Ma: Mater. Sci. Eng. A 381 (2004) 71-79.

2) M. A. Meyers, A. Mishra and D. J. Benson: Prog. Mater. Sci. 51 (2006) 427-556.

3) R. Z. Valiev and T. G. Langdon: Prog. Mater. Sci. 51 (2006) 881-981.

4) G. A. Malygin: Phys. Solid State 49 (2007) 1013-1033.

5) Q. Wei: J. Mater. Sci. 42 (2007) 1709-1727.

6) M. Dao, L. Lu, R. J. Asaro, J. T. M. De Hosson and E. Ma: Acta Mater. 55 (2007) 4041-4065.

7) E. O. Hall: Proc. Phys. Soc. B 64 (1951) 747-753.

8) N. J. Petch: J. Iron Steel Inst. 174 (1953) 25-28.

9) J. C. M. Li and Y. T. Chou: Metall. Trans. 1 (1970) 1145-1159.

10) R. W. Armstrong: Metall. Trans. 1 (1970) 1169-1176.

11) R. W. Armstrong: Yield, Flow and Fracture of Polycrystals, ed. by T. N. Baker, (Applied Science Pub., London, 1983) pp. 1-31.

12) N. Hansen: Metall. Trans. A 16 (1985) 2167-2190.

13) A. Lasalmonie and J. L. Strudel: J. Mater. Sci. 21 (1986) 1837-1852.

14) H. Conrad: Metall. Mater. Trans. A 35 (2004) 2681-2695.

15) C. S. Pande and K. R. Cooper: Prog. Mater. Sci. 54 (2009) 689-706.

16) R. W. Armstrong: Mechanical Properties of Nanocrystalline Materials, ed. by J. C. M. Li, Chap. 3, (Pan Stanford Pub., Singapore, 2011) pp. 61-91.

17) J. D. Eshelby, F. C. Frank and F. R. N. Nabarro: Philos. Mag. 42 (1951) 351-364.

18) J. Friedel: Dislocations, (Pergamon press, Oxford, 1964) pp. 260-263.

19) J. Weertman and J. R. Weertman: Elementary Dislocation Theory, (Oxford University Press, Inc., New York, 1992) pp. 126-130.

20) J. C. M. Li: Trans. Metall. Soc. AIME 277 (1963) 239-247.

21) R. W. Armstrong, I. Codd, R. M. Douthwaite and N. J. Petch: Philos. Mag. 7 (1962) 45-58.

22) J. Meakin and N. J. Petch: Report ASD-TDR-63-324, Symposium on the Role of Substructure in the Mechanical Behavior of Metals, (ASD7DR-63-324, Orlando, 1963) pp. 234-251.

23) H. Conrad: Electron Microscopy and Strength of Crystals, ed. by G. Thomas and J. Washburn, (Interscience, New York, 1963) pp. 299300 .

24) H. Conrad: Acta Metall. 11 (1963) 75-77.

25) H. Conrad, S. Feuerstein and L. Rice: Mater. Sci. Eng. 2 (1967) $157-$ 168.

26) U. F. Kocks: Metall. Trans. 1 (1970) 1123-1143.

27) A. W. Thompson, M. I. Baker and F. Flanagan: Acta Metall. 21 (1973) 1017-1028.

28) H. Margolin and M. S. Stanescu: Acta Metall. 23 (1975) 1411-1418.

29) M. A. Meyersm and E. Ashworth: Philos. Mag. A 46 (1982) 737-759.

30) M. F. Ashby: Philos. Mag. 21 (1970) 399-424.

31) N. Hansen: Yield, Flow and Fracture of Polycrystals, ed. by T. N. Baker, (Applied Science Pub., London, 1983) pp. 311-350.

32) N. Hansen: Acta Metall. 25 (1977) 863-869.
33) N. Hansen: Strength of Metals and Alloys, ed. by P. Haasen, (Pergamon Press, Oxford, 1979) pp. 849-854.

34) E. C. Aifantis: Int. J. Plasticity 3 (1987) 211-247.

35) N. A. Fleck and J. W. Hutchinson: J. Mech. Phys. Solids 41 (1993) 1825-1857.

36) N. A. Fleck, G. M. Muller, M. F. Ashby and J. W. Hutchinson: Acta Metall. Mater. 42 (1994) 475-487.

37) H. Gao, Y. Huang, W. D. Nix and J. W. Hutchinson: J. Mech. Phys. Solids 47 (1999) 1239-1263

38) H.-H. Fu, J. Benson and M. A. Meyers: Acta Mater. 49 (2001) 25672582.

39) L. Ma, J. Zhou, R. Zhu and S. Li: Mater. Sci. Eng. A 507 (2009) 42-49.

40) Y. V. R. K. Prasad and R. W. Armstrong: Philos. Mag. 29 (1974) 14211425 .

41) P. Rodriguez: Metall. Mater. Trans. A 35 (2004) 2697-2705.

42) R. W. Armstrong and P. Rodriguez: Philos. Mag. 86 (2006) 5787-5796.

43) H. Conrad and J. Narayan: Scr. Mater. 42 (2000) 1025-1030.

44) H. Conrad and J. Narayan: Acta Mater. 50 (2002) 5067-5078.

45) H. Conrad: Mater. Sci. Eng. A 341 (2003) 216-228.

46) H. Conrad and K. Jung: Mater. Sci. Eng. A 391 (2005) 272-284

47) H. Conrad and K. Jung: Mater. Sci. Eng. A 406 (2005) 78-85.

48) H. Van Swygenhoven, M. Spaczer and A. Caro: Acta Mater. 47 (1999) 3117-3126.

49) V. Yamakov, D. Wolf, M. Salazar, S. R. Phillpot and H. Gleiter: Acta Mater. 49 (2001) 2713-2722.

50) R. J. Asaro, P. Krysl and B. Kad: Phil. Mag. Lett. 83 (2003) 733-743.

51) K. S. Kumar, S. Suresh, M. F. Chisholm, J. A. Horton and P. Wang: Acta Mater. 51 (2003) 387-405.

52) S. Cheng, J. A. Spencer and W. W. Milligan: Acta Mater. 51 (2003) 4505-4518.

53) Y. M. Wang and E. Ma: Mater. Sci. Eng. A 375-377 (2004) 46-52.

54) R. W. Hayes, D. Witkin, F. Zhou and E. J. Lavernia: Acta Mater. 52 (2004) 4259-4271.

55) R. J. Asaro and S. Suresh: Acta Mater. 53 (2005) 3369-3382.

56) Y. M. Wang, A. V. Hamza and E. Ma: Acta Mater. 54 (2006) 27152726.

57) M. Kato, T. Fujii and S. Onaka: Mater. Trans. 49 (2008) 1278-1283.

58) M. Kato: Mater. Sci. Eng. A 516 (2009) 276-282.

59) C. Youngdahl, J. Weertman and R. C. Hugo: Scr. Mater. 44 (2001) 1475-1478.

60) G. Saada: Mater. Sci. Eng. A 400-401 (2005) 146-149.

61) H. Van Swygenhoven, P. M. Derlet and A. G. Froseth: Acta Mater. 54 (2006) 1975-1983.

62) H. Van Swygenhoven and J. R. Weertman: Mater. Today 9 (2006) 2431.

63) E. Ma, T. D. Shen and X. L. Wu: Nature Mater. 5 (2006) 841.

64) T. Kunimine, N. Takata, N. Tsuji, T. Fujii, M. Kato and S. Onaka: Mater. Trans. 50 (2009) 64-69.

65) T. Kunimine, T. Aragaki, T. Fujii, S. Onaka and M. Kato: J. Mater. Sci. 46 (2011) 4302-4307.

66) N. J. Karanjgaokar, C.-S. Oh, J. Lambros and I. Chasiotis: Acta Mater. 60 (2012) 5352-5361.

67) Y. Miyajima, H. Abe, T. Fujii, S. Onaka and M. Kato: Acta Mater. 61 (2013) 1537-1544

68) H. Conrad and D. Yang: J. Electron. Mater. 31 (2002) 304-312.

69) Y. R. Kapoor and J. K. Chakravartty: Acta Mater. 55 (2007) 54085418.

70) R. W. Armstrong: Mater. Trans. 55 (2014) 2-12. 